\title{
Das Dekorationsprogramm der königlichen Sonnenheiligtümer des Neuen Reiches nach einer Fassung der Spätzeit*
}

Wie die anderen Räume des Gebäudes des Taharqa hat auch die Dekoration von Kammer D sehr gelitten. Doch reichen die erhaltenen Reste aus, um einen Eindruck von der bemerkenswerten Komposition des Dekorationsprogrammes zu bekommen. Das außergewöhnliche Interesse dieses Programms an Szenen und Bildern liegt darin, daß uns hier der Grundbestand des Dekorationsprogramms eines Sonnenheiligtums des Neuen Reiches überliefert ist, der die Tradition der Sonnenheiligtümer von Der el Bahari und Medinet Habu fortsetzt und auf willkommene Weise ergänzt.

\section{1. Übersicht}

\begin{tabular}{|c|c|c|c|c|}
\hline $\begin{array}{l}\text { Anbringungs- } \\
\text { ort }\end{array}$ & Publikation & Szene Nr., Thema & Darstellung & Text \\
\hline $\begin{array}{l}\text { Südwand, } \\
\text { links }\end{array}$ & $\begin{array}{l}\text { S. } 37-38 \\
\text { Tf. } 18 \text { A }\end{array}$ & $\begin{array}{l}\text { 1: Anbetung des Son- } \\
\text { nengottes am Morgen }\end{array}$ & $\begin{array}{l}\text { Kg. in Begleitung von } \\
2 \times 4 \text { Pavianen über- } \\
\text { einander }\end{array}$ & $\begin{array}{l}\text { Text } 1 \text { (unidentifizier- } \\
\text { barer Sonnenhymnus) }\end{array}$ \\
\hline- , rechts & $\begin{array}{l}\text { S. } 38-40 \\
\text { Tf. } 18 \text { B, } \\
31-33\end{array}$ & $\begin{array}{l}\text { 2: Opfer zum } \\
\text { Sonnenaufgang }\end{array}$ & $\begin{array}{l}\text { Kg. vor Opfertisch, } \\
\text { im Rufgestus }\end{array}$ & $\begin{array}{l}\text { Text } 2 \text { (kulttheo- } \\
\text { logischer Traktat zum } \\
\text { Sonnenaufgang) }\end{array}$ \\
\hline Westwand & $\begin{array}{l}\text { S. } 40-41 \text {, } \\
\text { Tf. } 19\end{array}$ & $\begin{array}{l}\text { 3: Anbetung der } \\
\text { Abendbarke }\end{array}$ & $\begin{array}{l}\text { a) } 4 \times 3 \text { knieende } \\
\text { Götter, anbetend } \\
\text { b) oberer Teil : zerstört } \\
\text { unterer Teil: } \\
\text { Sonnenbarke }\end{array}$ & $\begin{array}{l}\text { a) Text } 3 \text { a (kosmo- } \\
\text { graphischer Text zum } \\
\text { Sonnenuntergang, } \\
\text { Tf. } 34 \text { ) } \\
\text { b) (ganz rechts:) } \\
\text { Text } 3 \text { b (id.) }\end{array}$ \\
\hline $\begin{array}{l}\text { Nordwand, } \\
\text { links }\end{array}$ & $\begin{array}{l}\text { S. } 42, \\
\text { Tf. } 20 \text { A }\end{array}$ & $\begin{array}{l}\text { 4: Opfer zum } \\
\text { Sonnenuntergang }\end{array}$ & entspricht Szene 2 & $\begin{array}{l}\text { Text } 4 \text { (kulttheo- } \\
\text { logischer Traktat zum } \\
\text { Sonnenuntergang) }\end{array}$ \\
\hline- , rechts & $\begin{array}{l}\text { S. } 43-46 \text {, Tf. } \\
20 \text { B, } 35-37\end{array}$ & $\begin{array}{l}\text { 5: Anbetung des Son- } \\
\text { nengottes am Abend }\end{array}$ & $\begin{array}{l}\text { Kg. knieend, anbetend, } \\
\text { mit Sonnenbarke }\end{array}$ & $\begin{array}{l}\text { Text } 5 \text { (Hymnus zum } \\
\text { Sonnenuntergang) }\end{array}$ \\
\hline $\begin{array}{l}\text { Ostwand, } \\
\text { links }\end{array}$ & $\begin{array}{l}\text { S. } 46-48 \text {, } \\
\text { Tf. } 21\end{array}$ & 6: Sonnenaufgang & $\begin{array}{l}\text { a) oben: Huh und } \\
\text { Hauhet, knieend mit } \\
\text { Sonnenkind und Ska- } \\
\text { rabäus } \\
\text { b) darunter: Isis und } \\
\text { Nephthys, Toeris u.A. }\end{array}$ & $\begin{array}{l}\text { Text } 6 \text { (Beischrift zur } \\
\text { Szene) }\end{array}$ \\
\hline,- rechts & ibd. & $\begin{array}{l}\text { 7: Anbetung der } \\
\text { Morgenbarke }\end{array}$ & $\begin{array}{l}\text { Sonnenbarke, von } \\
\text { Pavianen angebetet }\end{array}$ & $\begin{array}{l}\text { Text } 7 \text { (kosmographi- } \\
\text { scher Text über die } \\
\text { Paviane) }\end{array}$ \\
\hline
\end{tabular}

* Der Aufsatz war ursprünglich als Beitrag zu einer Rezension St. Wenigs von R. Parker - J. Leclant - J.-C. Goyon, The Edifice of Taharqa by the Sacred Lake of Karnak (Providence u. London 1979) gedacht. Zitate in vorliegendem Aufsatz mit Seiten- und Tafelangabe beziehen sich auf diese Publikation. 
Schon bei dieser Gliederung springt die paarweise antithetische Zuordnung der Szenen und Texte in die Augen:

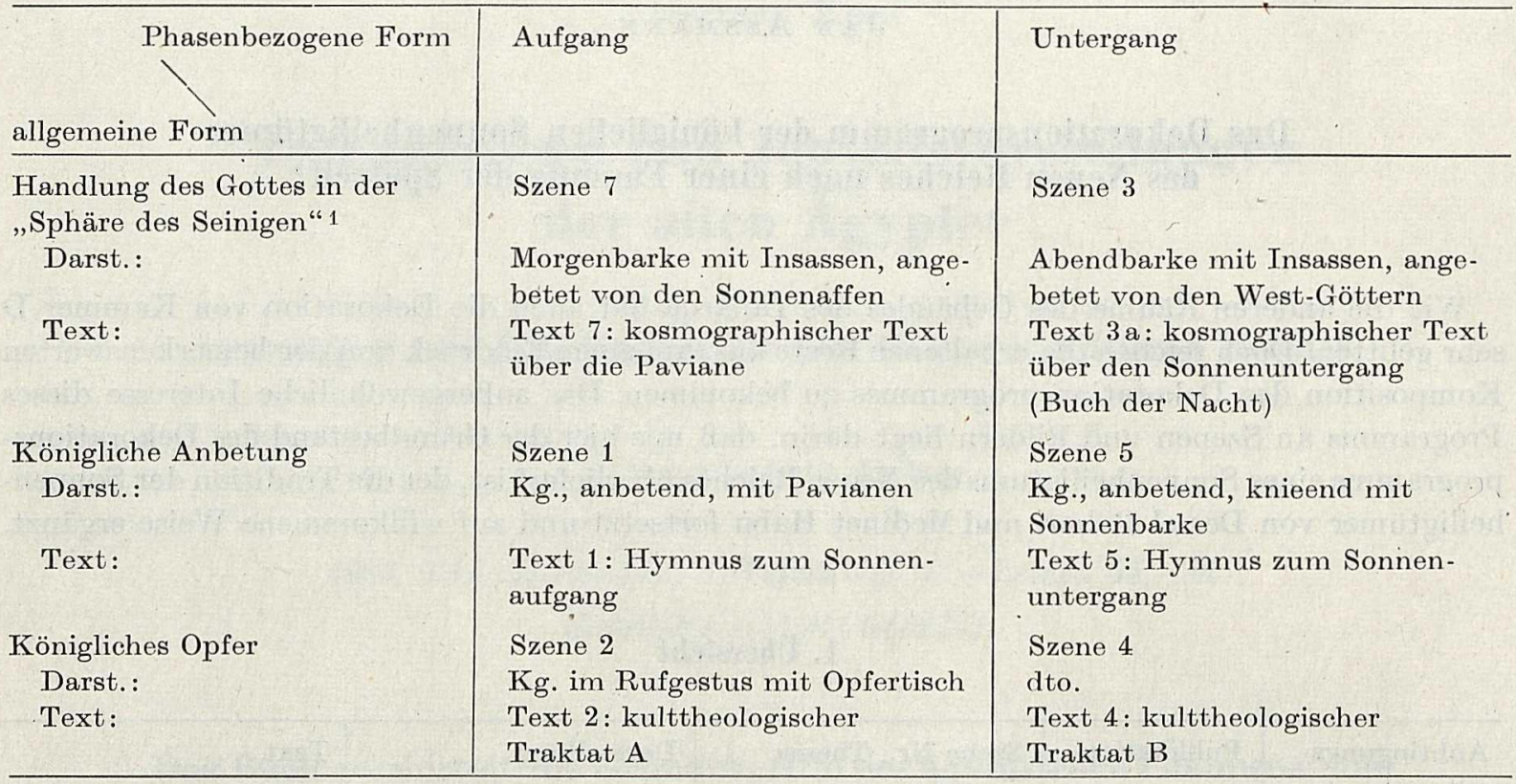

Wie man sieht, fällt nur Szene 6 (Sonnenaufgang) aus diesem Parallelismus heraus. Das läßt sich leicht erklären: Szene 6 hat die Position und erfüllt die Funktion eines „tableau final“, das in einem einzigen Bilde sowohl den gesamten Sonnenzyklus als auch die spezifische Phase des Sonnenaufgangs zum Ausdruck bringt ${ }^{2}$ und aufgrund dieser zusammenfassenden Sonderstellung kein Pendant zu haben braucht ${ }^{3}$.

\section{Einzelbemerkungen}

2.1 Szene 1. Die Darstellung hat, worauf Goyon hinweist, exakte Parallelen in Medinet Habu 4 . Vom Hymnus ist leider zu wenig erhalten, umihn mit einem anderenorts bezeugten Text identifizieren zu können.

2.2 Szene 2. Diese Szene kommt zusammen mit dem dazugehörigen Text nicht nur, worauf Goyon hinweist, in Medinet Habu vor ${ }^{5}$, sondern, was ihm entgangen ist, bereits im Sonnenheiligtum der Hatschepsut in Der el-Bahari ${ }^{6}$ und in Raum XVII des Luxor-Tempels Amenophis' III 7 . Text 2, dem ich 1970 eine Monographie gewidmet habe ${ }^{8}$, ist kein „Hymnus“, da er

$1 \mathrm{Zu}$ diesem Begriff vgl. meine Liturgischen Lieder, $333 \mathrm{ff}$.

2 Das ,tableau final“ des Pfortenbuchs ist nur das bekannteste Beispiel jener komplexen SonnenlaufBilder, die K. Sethe, Altägyptische Vorstellungen vom Lauf der Sonne, SBAW 1928, 259-284, sowie H. Schäfer, in: ZÄS 71 (1935) und Weltgebäude der alten Ägypter (1928) 83-128 behandelt haben. Vgl. neuerdings auch E. Hornung, in: MDIK 37 (1981), 217-126.

3 Allerdings bietet der zerstörte Tèil der Westwand genug Raum für eine Pendant-Szene in der Art von $\mathrm{MH} 423 \mathrm{C}$, links.

4 S. 37 n. 7 : MH VI 421 A, 424 C.

5 S. 38 n. 15, C. In MH ist die Darstellung zerstört. S. ausführlich J. Assmann, Der König als Sonnenpriester. Ein kosmographischer Begleittext zur kultischen Sonnenhymnik in thebanischen Tempeln und Gräbern (ADAIK 7, 1970), 7f., im Folgenden als KaS zitiert.

${ }^{6} \mathrm{KaS}, 10-14$; J. Karkowski, in: Etudes et travaux IX (1976), 73-77; Ders., in: Et. et trav. XI (1978), 217-219.

$7 \mathrm{KaS}, 3-6$ und passim; H. Brunner, Die südlichen Räume des Tempels von Luxor (AV 18, 1977), Tf. 65, S. 42 und $80-82$.

${ }^{8} \mathrm{KaS}$, von Goy on zwar gelegentlich zitiert (39 n. 21) aber nicht berücksichtigt. 
keinen Sprecher hat, sondern ein beschreibender Text, der vom Wissen des in die „Mysterien“ des Sonnenlaufs eingeweihten Königs als Priesters des Sonnengottes handelt. Er „begleitet" die hymnische Anbetung des Sonnengottes, indem er das Wissen als Bedingung ihrer Möglichkeit herausstellt. Ich möchte ihn einen „kulttheologischen Traktat" nennen; zusammen mit seinen Pendant Text 4 ist er gattungsmäßig (zumindest derzeit noch) ein Unikum. Jedenfalls kann man diesen für die Königs- und Sonnentheologie des Neuen und vermutlich zumindest auch des Mittleren Reichs ${ }^{9}$ außerordentlich wichtigen Text kaum irreführender rubrizieren denn als „Totenbuch 15 B“ 10 . Wenn er sich einmal unter die Sonnenhymnen des Totenbuchs eines königlichen Oberarchivars gesellt hat ${ }^{11}$, dann besagt das etwas über die mit dieser Stellung verbundenen Möglichkeiten, sich Zugang zu einem exklusiven Wissensvorrat zu verschaffen, aber gar nichts über Bestimmung und Herkunft des Textes. Mit dem Totenbuch hat er nichts zu tun. Von Text 2 sind derzeit $11 \mathrm{Be}-$ zeugungen bekannt: 3 aus königlichen Sonnenkultstätten des NR ${ }^{12}, 3$ auf königlichen Denkmälern der Äthiopendynastie ${ }^{13}, 3$ in Privatgräbern der Saitenzeit, die auch sonst kgl. Texte des NR reproduzieren ${ }^{14}$, und nur 2 auf Privatdenkmälern der 20. Dyn., eine in dem erwähnten Totenbuch, die andere, eine Kurzfassung, im Grab des 3. Amunspropheten Tjanefer (TT 158) ${ }^{15}$, das u. a. auch Auszüge aus dem Pfortenbuch enthält ${ }^{16}$. Wir haben es also eindeutig mit einem Text zu tun, der zu demselben exklusiven Wissensvorrat gehört wie die Unterweltsbücher und sonstigen liturgischkosmographischen Kompositionen der Königsgräber des Neuen Reichs ${ }^{17}$.

2.3 Szene 3 ist' am stärksten zerstört. Genaue Parallelen scheinen nicht nachweisbar. Die $4 \times 3$ Knieenden des linken Abschnitts entsprechen den $4 \times 3$ ntrw jmntjw, die $\mathrm{MH}$ in gleicher Haltung, aber nicht über- sondern nebeneinander darstellt ${ }^{18}$. In der (unter Echnaton getilgten und später restaurierten) Luxor-Fassung, die Goyon nicht kannte, sind sie stehend dargestellt ${ }^{19}$. Der rechte Teil der Szene enthält die von den Westgöttern angebetete und nach Goyons überzeugender Ergänzung von 4 Schakalen getreidelte Sonnenbarke mit viel Platz darüber. Eine Ergänzung im Sinne von MH VI $422 \mathrm{C}^{20}$ wird von Goyon nicht in Erwägung gezogen.

Text $3 \mathrm{a}$ ist, von Goyon identifiziert, der Text zur 1. sbht aus dem Buch der Nacht 21, der außer-

\footnotetext{
$9 \mathrm{KaS}, 5$ n. 1.

10 passim, z. B. 37 n. 3,38 n. 15 .

11 pBM 9953 B s. KaS 14. Der folgende Sonnenhymnus ist als Paralleltext zu p Berlin 3050 Text "A“ ebenfalls liturgisch und entstammt demselben exklusiven Wissensvorrat wie der Traktat, s. Lit. Lieder, $187 \mathrm{ff}$.

12 Der el Bahari s. n. 6; Luxor s. n. 7; Medinet Habu s. n. 5.

13 Außer Taharqa noch die beiden von Goyon herangezogenen Särge aus Nuri: S. 38 n. 15, A und B.

${ }_{14}$ TT 33 s. KaS, $15 \mathrm{f}$; TT 27 s. A. Roceati, in: OA 15 (1976) 234 n. 4; Saqqara s. E. Bresciani, in: Egitto e Vicino Oriente 1 (1978) Tf. XVIII.

$15 \mathrm{KaS}, 15$. Lies bei Goyon, 38 n. $15 \mathrm{D}$ und Tf. 31 „158“ statt „148“.

16 K. C. Seele, The Tomb of Tjanefer, Tf. $32 \mathrm{ff}$. Beachte, daß sich die Bezeichnung „Book of Gates“ bei Porter-Moss auf einen ganz anderen Zyklus bezieht. Die privaten Belege des königlichen Textes sind übrigens sorgfältig redigiert. So verändert z. B. pBM $9953 \mathrm{~B}$ den Satz $j w r d j . n R^{`} w \mathrm{~N} t p t 3 n$ ` $n h w$ „Re hat $\mathrm{N}$ auf der Erde der Lebenden eingesetzt" in : $j w$ wsjr $\mathrm{N} t p t 3 n$ " $n h w$ „Osiris $\mathrm{N}$ ist auf der Erde der Lebenden", doch offenbar, um die allzu spezifisch königliche Idee eines göttlichen Eingesetztseins zu vermeiden. TT 158 ist eine Kurzfassung, die sich überhaupt auf den Anfang beschränkt.

$17 \mathrm{Zu}$ Goyons Übersetzung S. 38-40 möchte ich folgendes anmerken:

a) „King $\mathrm{N}$ has worshipped ..." : die $j w \mathrm{~N} s \underline{d m}$. $f$-Form bezieht sich nicht auf ein aktuelles Ereignis im perfektischen Sinne, sondern auf eine allgemeine Disposition, vgl. KaS, 59 n. 3.

b) „he is in peace ..."

c) "to the living": Übersetzung berücksichtigt nicht das überall davorstehende $t p t 3$. Die in n. 36 gegebene Alternative ist richtig.

d) „the living who depend upon him": lies " $n h . f$ „he lives"; die Schreibung von ' $n h$ mit 3 Pluralstrichen ist eine den Äthiopensärgen eigentümliche Abweichung.

$18 \mathrm{KaS}, 9$ n. 2; Goyon, 40 n. 2.

19 Brunner, a. a. O., Tf. 41 links.

${ }^{20}$ Isis und Nephthys erheben knieend den geflügelten Skarabäus.

${ }^{21}$ A. Piankoff, Le livre du jour et de la nuit (BE 13), 35.
} 
halb dieses Buches nicht nur in Medinet Habu ${ }^{22}$, sondern bereits in Der el Bahari vorkommt ${ }^{23}$. Text 3 b, von Goyon mit MH VI 422 B 26-33, der Fortsetzung des Textes aus dem Buch der Nacht, identifiziert, hat weder im Buch der Nacht, noch in Der el Bahari eine Entsprechung und zeigt, daß Taharqa eine speziell Medinet Habu nahestehende Vorlage benutzt hat.

2.4 Szene 4 entspricht genau Szene 2 und stellt das abendliche Opfer zum Sonnenuntergang dar. Wiederum ist der zugehörige Text 4 kein „Hymnus“, sondern ein beschreibender Text, der die kosmisch-götterweltlichen Vorgänge beim Sonnenuntergang und die Bedingungen der Möglichkeit ihrer kultischen Begleitung behandelt. Parallelen finden sich in Der el Bahari ${ }^{24}$ und in Luxor, ebenfalls als abendliches Pendant zu Text $2{ }^{25}$. In Luxor ist auch dieser Text durch gegenläufige Kopie einer rückläufig geschriebenen Vorlage verunklärt. Leider reicht der bei Taharqa erhaltene Textbestand nicht aus, um den Luxor-Text wiederherzustellen. In Verbindung mit den unpublizierten Fragmenten von Der el Bahari müßte sich dieses Problem jedoch lösen lassen. Eigenartigerweise fehlt dieser Text in Medinet Habu 26.

2.5 Szene 5 entspricht ziemlich genau MH VI 422 A. Dort betet der König im hnw-Gestus ${ }^{27}$, wie bei Taharqa auf einem Podest knieend. In $\mathrm{MH}$ ist jedoch ihm gegenüber, stehend mit Falkenkopf, der Sonnengott dargestellt, bei Taharqa die Sonnenbarke, die aber nicht ihm entgegen, sondern in gleicher Blickrichtung fährt. Der Hymnus kommt außer in Medinet Habu auch im Totenbuch der Königin Nedjemet vor ${ }^{28}$. Das genügt für Goyon wieder, ihn als „,chapter $15 \mathrm{D}$ of the Book of the Dead" zu erklären, „borrowed, as was chapter 15 B also (sc. Text 2, der kulttheologische Traktat zum Sonnenaufgang), for the sun liturgy" 29. Das Umgekehrte ist der Fall: Königin Nedjemet hat ihre Sonnenhymnen aus einer liturgischen Quelle entlehnt:

$\begin{array}{ll}\text { Zeile } 1 \mathrm{ff.} & =\text { MH VI } 420 \text { B } 1-3+424 \text { B } 4-11 \\ \text { Zeile } 18 \mathrm{ff.} & =\text { Stundenritual, } 7 \text {. Std. } \\ \text { Zeile } 25 \mathrm{ff.} & =\text { Liturg. Lieder Text II } 1 \\ \text { Zeile } 29-31 & =\text { Apopislitanei Lit. Lieder, } 200 \\ \text { Zeile } 47-50 & =\text { Text liturg. Charakters ÄHG Nr. } 19 \\ \text { Zeile } 51 \mathrm{ff.} & =\text { Text } 5\end{array}$

Dieser Text bringt die Grundprinzipien des Sonnenkults besonders klar zum Ausdruck: die Analogie zwischen den Vorgängen der götterweltlichen und der kultischen Sphäre. Der König preist den Gott mit genau den Worten, die ihm aus dem götterweltlichen Kreise der „Sphäre des Seinigen“

22 Ders., in : ASAE 42 (1943) $351 \mathrm{ff}$.

$23 \mathrm{KaS}, 11$; Karkowski, ET IX, $69 \mathrm{f}$.

${ }_{24} \mathrm{~J}$. Karkowski, ET IX, 80.

25 Brunner, a. a. O., Tf. 41 ; KaS, 5 n. 3 vgl. 8 f.

26 Entgegen der KaS 9 geäußerten Vermutung sehe ich auch keine Lïcke, in der er dort unterzubringen wäre. Einige Bemerkungen zum Text und seiner Übersetzung:

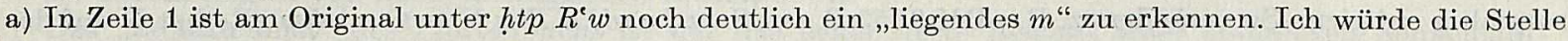
daher folgendermaßen rekonstruieren:

[...] $m$-Msktt

$h t p-R^{6} w m-[j r t] . f-w n m j t$

wn $[\ldots]$

Der Satz „Re nimmt Platz in seinem rechten [Auge]" bezieht sich entweder auf das Einsteigen in die dem rechten Auge gleichgesetzte Nachtbarke, oder auf das Eintreten des Gottes in die Sonnenscheibe; gerade in

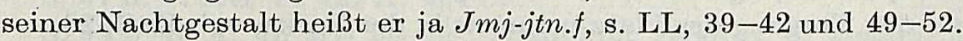

b) „have welcomed him those ..." setzt eine Textänderung voraus: šzp.n sn $>s ̌ z p . n$ sw. Eher: „nachdem die Unterweltlichen sie empfangen haben".

c) „... sailing to cross the east ...": die Luxor-Fassung liest $n^{e e} w r n m^{e} j m n t j$. Ob $n m^{e}$ das im Wb nicht belegte Wort für „Korridor" darstellt? Vgl. Meeks, Année Lexicographique 1 (1977), 193 Nr. 77. 2109.

27 Aufgrund der anderen Beinstellung lehnt Goyon, 43 n. 29 mit Recht eine derartige Ergänzung für Taharqa ab.

2844 n. 30 vgl. KaS 8 m. n. 5 und Lit. Lieder, 182 n. 69.

2943 , s. auch 44 n. 30 . 
gesagt werden (und in die der König kraft seines besonderen Wissens eingeweiht ist ${ }^{30}$ ) und der Gott wendet sich dem König genauso zu, wie er sich den Mitgliedern seiner götterweltlichen Eigensphäre zuwendet.

2.6 Für Szene 6, die Geburt der Sonne am Morgen, kann Goyon auf der Basis der Parallele in Medinet Habu ${ }^{31}$ eine Ergänzung vorschlagen, die - wie eine vollständige, unpublizierte Fassung dieser Szene im Grab des Ibi zeigt ${ }^{32}$ - im wesentlichen richtig ist. Das Tableau ist zweigeteilt. Im oberen Teil sitzen Huh und Hauhet einander gegenüber und heben die Sonne in Form des „,sitzenden Kindes“ hoch; darüber spannt sich der geflügelte Skarabäus. Im unteren Teil stehen Isis und Nephthys und erheben die Sonne in Gestalt der Scheibe. Die beiden Elemente der Szene finden sich in anderer Anordnung auch im Buch des Tages ${ }^{33}$. Die begleitenden Texte sind bei Ibi wesentlich ausführlicher. Aufgrund der Anwesenheit von Huh und Hauhet bezieht Goyon diese Darstellung auf "the hermopolitan myth". Huh und Hauhet werden aber in gleicher Funktion auch im „Traktat“ (Text 2) erwähnt sowie im Amduat, Buch vom Tage usw. ${ }^{34}$, einer Literatur, die gewiß heliopolitanischen Ursprungs ist. Hier haben wir es sicher nicht mit einem lokal gebundenen Mythos, sondern mit allgemein-ägyptischer Kosmologie zu tun ${ }^{35}$.

2.7 Der „Paviantext“ (Text 7) unter der Sonnenbarke, in Medinet Habu verdoppelt zu beiden Seiten der Sonnengeburtsszene angebracht ${ }^{36}$, schließt hier in einfacher Form rechts an die Geburtsszene an. Die Verdopplung in Medinet Habu läßt darauf schließen, daß hier beide Barken, die des Tages und die der Nacht dargestellt waren ${ }^{37}$. Bei Taharqa beschränkt sich die Darstellung auf die rechte Barke, die Goyon aus nicht ganz einsichtigen Gründen als Nachtbarke ergänzt ${ }^{38}$. Text 7 ist außer in MH und auf dem Sarg des Aspelta auch bereits in Der el Bahari bezeugt, dort in antithetischem Parallelismus mit Text $3 \mathrm{a}^{39}$. Auch dort bezieht sich der Text auf die 6 . Stunde der Nacht, zugleich aber auf die Vorgänge des Sonnenaufgangs ${ }^{40}$.

\section{Zusammenfassung}

Das Dekorationsprogramm von Kammer D ist kein Sammelsurium, das aus verschiedenen Quellen zusammengetragen wurde, sondern realisiert einen zusammengehörigen Zyklus von liturgischen (Texte 1,5), kosmographischen (Texte 3, 7) und kulttheologischen Elementen

30 Dies ist das Thema von Text 2, vgl. KaS, $56 \mathrm{f}$.

31 S. 47 mit Verweis auf MH VI 420 B, Mitte, wiedergegeben auf Tf. 16 D.

32 Sargkammer, Rückwand; K. P. Kuhlmann, W. Schenkel, in: MDIK 28 (1973) 209, 2. (4).

33 S. 47 n. 30 mit Verweis auf Piankoff, BE 13 Tf. VIII und einen Sarg im Louvre, der aber lediglich eine weitere Version des Buchs des Tages und der Nacht darstellt. Näher kommt Mariette, Monuments divers, Tf. 46. Hier treten tatsächlich auch die übrigen Mitglieder der hermupolitanischen Achtheit als Adoranten des Sonnengottes auf.

34 Vgl. KaS, 42-47, 42 n. 2.

35 Es scheint sich um einen jener Fälle zu handeln, in denen die „kultische Heimat“ einer Gottheit nicht zu ihrem ursprünglichen Wesen gehört, sondern das Ergebnis eines sekundären kulttopographischen Ansiedlungsprozesses darstellt; vgl. E. Hornung, Der Eine und die Vielen (1971) 219-223. Zu „Hermupolis" vgl. n. 33. Auf S. 48 ist nach Zeile 7 Text ausgefallen.

36 MH VI 420 B, 1-12 und 13-24; KaS, 28 f., dort bereits im Sinne der von Goyon 46 n. 11 geäußerten Kritik an Lit. Lieder 208 n. 92 übersetzt.

37 Vgl. hierzu E. Thomas, in: JEA 42 (1958) 65-79.

38 Vielleicht weil rechts die Seite des Westens und der Nacht ist? Bei Aspalta ist aber die Tagbarke dargestellt.

39 Karkowski, ET IX, 70-72; KaS, 5.

40 Goy on überschreibt Szenen 6 und 7 als „Nocturnal Voyage and rebirth of the sun“ (S. 46), versteht also offenbar die über dem „Paviantext" angebrachte Barke als Darstellung der Nachtfahrt des Sonnengottes. Ich nehme dagegen an, daß hier die Tagbarke dargestelli, war, diese Darstellung sich auf den Satz htp.f $m$ $M^{\prime} n \underline{d} t$ in Text 2 und die gesamte Dekoration der Ostwand sich ausschließlich auf den Sonnenaufgang bezieht. 
(Texte 2,4). Mit Ausnahme der liturgischen Teile ist der Zyklus bereits in Der el-Bahari bezéugt und kommt dann, in seinem liturgischen Teil stark erweitert, auch in Medinet Habu vor:

Taharqa Der el Bahari Medinet Habu

Text 1

Text 2

Text $3 a+b$

Text 4

Text 5

Text 6

Text 7

$\begin{array}{ll}+ & + \\ 3 a & 3 a+b \\ + & - \\ -(?) & + \\ -(?) & + \\ + & +\end{array}$

Auf den Wänden der Kammer D ist dieser Zyklus so angebracht worden, daß sich die jeweils äquivalenten Elemente genau gegenüberstehen: die Kosmographien des Sonnenauf- (Szenen 6+7) und Sonnenuntergangs (Szene 3) auf der Ost- und Westwand, die Kulttheologie am Westende der Süd- (Text 2) und Nordwand (Text 4), die Liturgie (Hymnen) am Ostende der Süd- (Text 1) und Nordwand (Text 5) (s. Abb. 1)..

Medinet Habu:

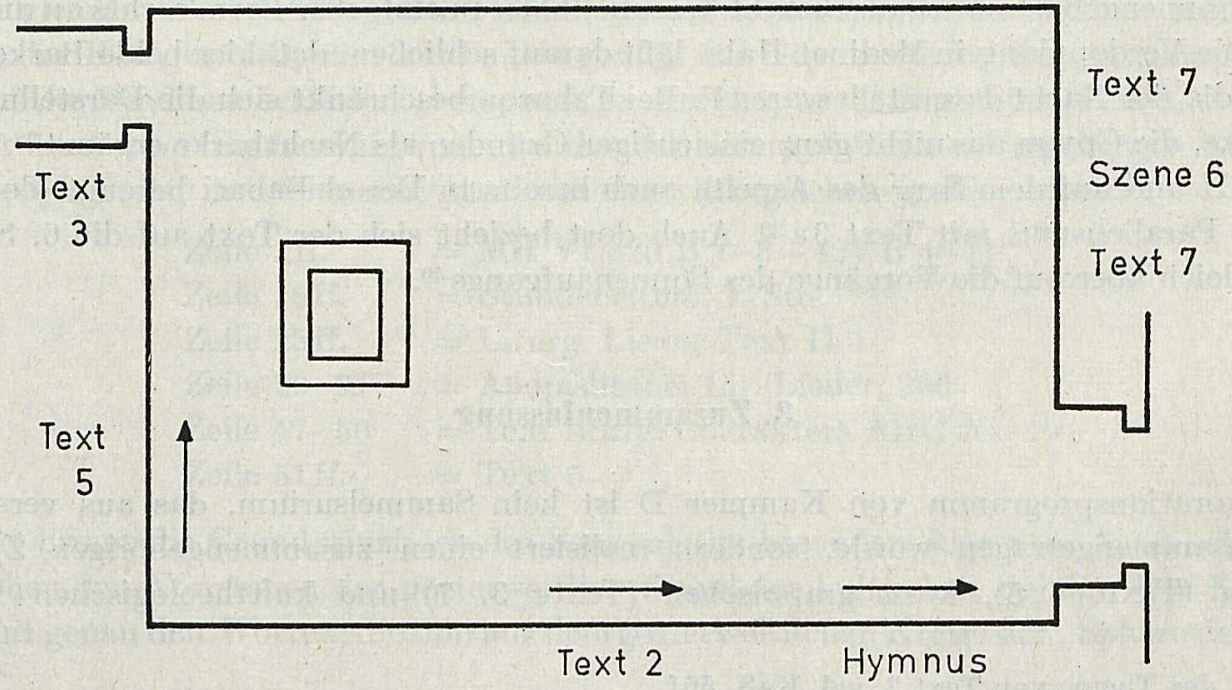

\section{Karnak}
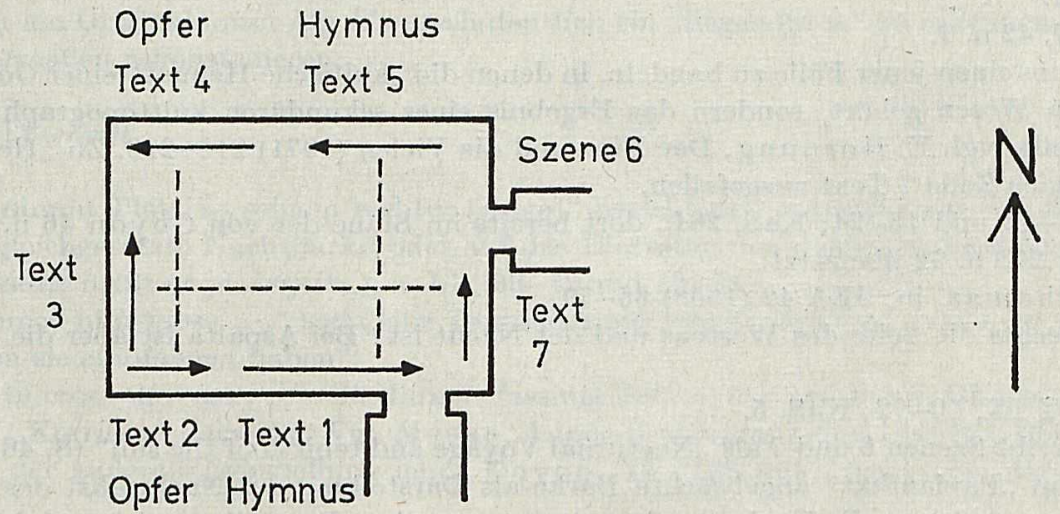

Abb. 1. Anordnung der parallelen Texte in Medinet Habu und in Karnak 
Die Verbindung von Kosmographie und Liturgie ist ein Spezifikum des Sonnenkults 41 . Das eindrucksvollste Beispiel bietet das Stundenritual, dessen kosmographisches Komplement das Buch vom Tage darstellt 42 .

Der ägyptische Sonnenkult versteht sich als die kultische Begleitung der kosmischen Vorgänge, die sich unter dem Begriff des „Sonnenlaufs" zusammenfassen lassen. Diese Begleitung hat eine doppelte Funktion: 1. die kosmischen Vorgänge mitwirkend zu fördern („der Sonnenbarke freie Fahrt zu verschaffen " $)^{43}$, 2. die irdische Ordnung in die kosmische einzubinden und kraft dieser Kohärenz die irdische Welt am kosmischen Leben (dessen Inbegriff der Sonnenlauf darstellt) teilhaben zu lassen ${ }^{41}$. Dieses voraussetzungsreiche Geschäft obliegt dem König ${ }^{45}$. Die wichtigste Voraussetzung ist das Wissen. Das in kosmische Zusammenhänge ausgreifende kultische Handeln des Königs basiert auf genauer Kenntnis dieser kosmischen Ebene ${ }^{46}$. Die kosmographische Literatur bewahrt und vermittelt das Wissen, das der König braucht, um den Sonnenlauf - und damit die Zeit, und die Wirklichkeit überhaupt - in Gang zu halten.

Wir haben es hier mit einem Wissensvorrat zu tun, der einerseits an den Sonnenkult, andererseits an den König gebunden ist. Die Könige des Neuen Reichs haben aus naheliegenden Gründen Teile dieses Wissensvorrazs zur Dekoration ihrer Felsgräber verwendet, daher verbindet sich in der Ägyptologie damit noch immer die Vorstellung von Totenliteratur. Seinen eigentlichen „Sitz im Leben" aber hat man im Sonnenkult zu suchen, dessen Zentrum, Heliopolis, so gut wie verloren ist, dessen Filialkulte wir aber in den Sonnenheiligtümern des NR zu erblicken haben, von denen uns wenigstens Reste erhalten sind ${ }^{47}$. Zu diesen Denkmälern gehört auch der Westteil des TaharqaGebäudes, die Treppe mit der Sonnenlitanei und die Kammer D. Die Texte und Bilder sind hier an ihrem eigentlichen Ort und nicht etwa aus disparaten anderen Bereichen, der privaten („Totenbuch 15") und königlichen (Sonnenlitanei, Buch der Nacht) Totenliteratur zusammengetragen. Vielmehr hat umgekehrt der Bereich des mit dem Sonnenkult verbundenen Wissensvorrats in andere Zusammenhänge, vor allem die königlichen Jenseitsvorstellungen, ausgestrahlt. Demgegenüber ist die Bezeugung derartiger Materialien in Privatdenkmälern vor der Spätzeit auffallend gering: daran erweist sich die Exklusivität dieses Wissensvorrats.

Einmal mehr zeigt sich, daß der in der Wissenschaft eingebürgerte Begriff „Totenbuch Kap. 15“ falsch ist und den Einblick in die wirklichen Zusammenhänge verhindert ${ }^{48}$. Wir sollten diesen Begriff auf das entsprechende Kapitel der Spätzeitfassung des Totenbuchs beschränken, auf das Lepsius ihn gemünzt hatte. Dieses Kapitel besteht aus 9 Einzeltexten und stellt so etwas wie ein Kompendium der Sonnenanbetung dar, wie es als Ergebnis eines langen Kanonisierungsprozesses aus der Fülle der im Neuen Reich vorkommenden Sonnenhymnen festgelegt worden war. Einzeltexte dieses Kompendiums lassen sich bereits im Neuen Reich belegen, und sogar Verbindungen wie $a-d^{49}$ und a-f ${ }^{50}$ kommen vor. Aber nicht einmal diese Fälle sind als „Totb 15 (a/b/c usw. " angemessen gekennzeichnet. Sie entsprechen dem späteren Kap. 15 (a/b/c usw.), aber sie sind es noch nicht. Sie haben keinen „kanonisierten“ Status gegenüber „freien“ Sonnenhymnen, sondern sie

\footnotetext{
41 S. hierzu KaS, passim.

42 Vgl. Liturg. Lieder, 113-164; $\ddot{H} H G$ Nr. 1-12. Neuedition durch E. Graefe in Vorbereitung.

43 S. hierzu Lit. Lieder, $211 \mathrm{ff}$; KaS, 68-69.

44 Vgl. Ph. Derchain, Le Pap. Salt 825, rituel pour la conservation de la vie en Egypte (1965) I, $4 \mathrm{ff}$.

45 Vgl. Ph. Derchain, Le rôle du roi d'Egypte dans le maintien de l'ordre cosmique, in: Le Pouvoir et le Sacré (1962).

46 Vgl. Derchain, le pap. Salt 825, I, 6: „lui seul était capable de concevoir en une fois la totalité de ce qui est, et il sentait vaguement que l'unité ressentie cesserait dès qu'il cesserait de la penser".

47 R. Stadelmann, in: MDIK 25 (1969) 159-178.

48 In dem allgemeinen (und irreführenden) Sinne, daß jeder in einem Totenbuch auftretende Sonnenhymnus als „Variante“ von „Kap. 15“ bezeichnet wird, wird der Begriff bei Naville, Allen und Barguet verwendet. Budge unterschied richtig zwischen ,introductory hymns" und „chapter 15“.

$49 \mathrm{Z}$ B. der Block des Obersten $R 3 j j$ in Brooklyn 37. $46 \mathrm{~L}$ und die Stele Vatican Nr. 251, beide frühe 19. Dyn.

${ }^{50}$ pBM 10470 (Ani), frühe 19. Dyn.
} 
sind genau so „frei“ wie jene. Im NR gehören Sonnenhymnen noch nicht zum kanonisierten Spruchkorpus des Totenbuchs, sondern werden ihm gleichsam vorgeschaltet, wobei die Schreiber in der Auswahl solcher Hymnen offenbar dieselbe Freiheit haben wie in der Beschriftung der Grabeingänge. Es empfiehlt sich daher, dreierlei zu unterscheiden: 1. eine „landläufige“ Sonnenhymnik, aus der derartige Hymnen geschöpft sind; dieses Phänomen scheint weitgehend auf das NR beschränkt, 2. eine „funeräre" Sonnenhymnik (Totenbuch 15) mit dem Status eines kanonisierten „Spruchs"; dies scheint eine Besonderheit der Spätzeit und 3. eine liturgische Sonnenhymnik, die im Kult der Sonnenheiligtümer verankert war und zum selben exklusiven Wissensvorrat gehört wie die in den Königsgräbern aufgezeichneten Kosmographien; diese Texte schwer bestimmbaren Alters reichen z. T. sicher weit vor das NR zurück, erweitern ihren Bestand im NR und werden im Rahmen einer reproduktiven und kanonisierten Überlieferung bis in die Spätzeit tradiert. Diesem Bereich, der sich uns erst durch die Forschungen der letzten Jahre, vor allem an den Gräbern der Saitenzeit, näher zu erschließen beginnt, gehören die Texte von Kammer D an. Derartige Distinktionen mögen pedantisch erscheinen. Was ändert sich an unserem Verständnis eines Textes, wenn wir ihn als „Totenbuch 15 B“ bezeichnen oder nicht? Tatsächlich hängt unser Verständnis eines Textes auch davon ab, daß er sich dem spezifischen Bereich kulturellen Wissens und Handelns zuordnen läßt, in dem er ursprünglich verankert war ${ }^{51}$. Wenn wir, wie bei religiösen Texten üblich, alles in einen Topf werfen - „Totenbuch $15^{\text {" }}$ - finden wir uns Vorstellungswelten von einer Komplexität konfrontiert, die jedes Verstehen ausschließt. Umgekehrt zerreißen wir eine geschlosséne Einheit, wenn wir die Elemente verschiedenen Bereichen wie dem Totenbuch und dem „hermopolitanischen Mythos" usw. zuweisen.

Daß diese allgemeinen Bemerkungen nicht dem verdienstvollen Herausgeber der Taharqa-Texte und Darstellungen am Zeuge flicken wollen, bedarf keines Wortes. Sie wollen vielmehr dazu beitragen, das Interesse der von J. C. Goyon gewissenhaft und kenntnisreich edierten und kommentierten Texte und Darstellungen ins rechte Licht zu rücken.

51 Niemand hat das deutlicher gezeigt als J. Goy on selbst in seinem Aufsatz über ,la véritable attribution des soi-disants chapitres 191 et 192 du Livre des Morts", in: Studia Aegyptiaca I (1974) 117-127. 